\title{
A national evaluation of geographic accessibility and provider availability of obesity medicine diplomates in the United States between 2011 and 2019
}

\author{
Catherine C. Pollack $\mathbb{D i D}^{1,2^{凶}}$, Tracy Onega ${ }^{3}$, Jennifer A. Emond ${ }^{1,4}$, Soroush Vosoughi ${ }^{5}$, A. James O'Malley ${ }^{1,6}$, Auden C. McClure ${ }^{4,6,7}$, \\ Richard I. Rothstein ${ }^{7,8}$ and Diane Gilbert-Diamond ${ }^{2,4,7,8}$
}

(c) The Author(s), under exclusive licence to Springer Nature Limited 2021

\begin{abstract}
BACKGROUND/OBJECTIVES: Obesity is a pressing health concern within the United States (US). Obesity medicine "diplomates" receive specialized training, yet it is unclear if their accessibility and availability adequately serves the need. The purpose of this research was to understand how accessibility has evolved over time and assess the practicality of serving an estimated patient population with the current distribution and quantity of diplomates.

METHODS: Population-weighted Census tracts in US counties were mapped to the nearest facility on a road network with at least one diplomate who specialized in adult (including geriatric) care between 2011 and 2019. The median travel time for all Census tracts within a county represented the primary geographic access measure. Availability was assessed by estimating the number of diplomates per 100000 patients with obesity and the number of facilities able to serve assigned patients under three clinical guidelines.

RESULTS: Of the 3371 diplomates certified since 2019, 3036 were included. The median travel time (weighted for county population) fell from 28.5 min [IQR: 13.7, 68.1] in 2011 to $9.95 \mathrm{~min}$ [IQR: 7.49, 18.1] in 2019. There were distinct intra- and inter-year travel time variations by race, ethnicity, education, median household income, rurality, and Census region (all $P<0.001$ ). The median number of diplomates per 100000 with obesity grew from 1 [IQR: 0.39, 1.59] in 2011 to 5 [IQR: 2.74, 11.4] in 2019. In 2019, an estimated 1.7\% of facilities could meet the recommended number of visits for all mapped patients with obesity, up from $0 \%$ in 2011 .

CONCLUSIONS: Diplomate geographic access and availability have improved over time, yet there is still not a high enough supply to serve the potential patient demand. Future studies should quantify patient-level associations between travel time and health outcomes, including whether the number of available diplomates impacts utilization.
\end{abstract}

International Journal of Obesity (2022) 46:669-675; https://doi.org/10.1038/s41366-021-01024-9

\section{INTRODUCTION}

The prevalence of adult obesity in the United States (US) now exceeds $40 \%$, and patients with obesity are at higher risk for various diseases including type 2 diabetes, cardiovascular disease, cancers, and the novel coronavirus disease (COVID-19), among others [1-5]. Yet, key facets of obesity medicine such as the basic disease pathophysiology, prevention methodologies, and best practices for working with patients with obesity may be underrepresented within the medical school curriculum [6-8]. This is further evident in board certification exams, the majority of which only mention related terminology and not obesity itself [9]. The American Board of Obesity Medicine (ABOM) provides an additional certification pathway for clinicians beyond traditional medical training through either $60 \mathrm{~h}$ of continuing medical education (CME) credits or an obesity medicine fellowship with at least $500 \mathrm{~h}$ of obesity-specific training $[10,11]$. Both pathways require an exam that includes topics such as the "basic concepts" of obesity (including determinants and pathophysiology); diagnostic and evaluation metrics (including physical assessments and laboratory testing); treatment modalities (including pharmacotherapies, surgical procedures, and behavioral therapies); and "practice management strategies" (including the mitigation of weight bias and cost variations in treatment options) [12]. By undergoing this additional training, clinicians ensure that they are well-equipped to provide patients with meaningful, cutting-edge treatment regardless of medical specialty [13]. While the ABOM was only created in 2011, studies have already shown that obesity medicine "diplomates" are better equipped than are general practitioners to recognize the complex causes of and guide medical and surgical treatments for obesity $[14,15]$.

\footnotetext{
${ }^{1}$ Department of Biomedical Data Science, Geisel School of Medicine at Dartmouth, Hanover, NH, USA. ${ }^{2}$ Department of Epidemiology, Geisel School of Medicine at Dartmouth, Hanover, NH, USA. ${ }^{3}$ Department of Population Health Sciences, Huntsman Cancer Institute, University of Utah, Salt Lake City, UT, USA. ${ }^{4}$ Department of Pediatrics, Geisel School of Medicine at Dartmouth, Hanover, NH, USA. ${ }^{5}$ Department of Computer Science, Dartmouth College, Hanover, NH, USA. ${ }^{6}$ The Dartmouth Institute for Health Policy and Clinical Practice, Hanover, NH, USA. ${ }^{7}$ Dartmouth-Hitchcock Weight and Wellness Center, Department of Medicine at Dartmouth-Hitchcock Medical Center, Lebanon, NH, USA. ${ }^{8}$ Department of Medicine, Geisel School of Medicine at Dartmouth, Hanover, NH, USA. ${ }^{凶}$ email: Catherine.c.pollack.gr@dartmouth.edu
} 
A previous state-level, cross-sectional analysis in 2019 compared the number of diplomates with the prevalence of obesity, finding a significantly higher number practiced in southern states compared to western states, mirroring geospatial variations in obesity prevalence [16]. However, there exists a need to perform localized analysis to have a better understanding of whether patient populations in need can access the care that they require. Thus, this study aimed to expand prior literature by identifying geographic patterns in the distribution of diplomates, understand how the accessibility of these diplomates has changed since the creation of the ABOM certification program, and assess whether current availability is sufficient to match an estimated population with obesity.

\section{METHODS \\ Diplomate identification and location mapping}

To evaluate changes in the physical and practical accessibility of all obesity medicine diplomates within the US between 2011 and 2019, the practicing location for all diplomates was determined. Obesity diplomates were identified through the "Diplomate Search and Certification Verification" portion of the ABOM website, which includes diplomates' names, specialty, date of certification, and general location (city and state) [17]. A comprehensive web scraper was developed to automatically curate the list of obesity diplomates and identify the corresponding practicing facilities (a complete description of this process is available in eMethods 1). After list compilation, addresses were geocoded with Google Maps to obtain each facility's precise latitude and longitude. It was assumed that a diplomate had remained at the listed practicing facility since their certification. Diplomates with a specialty in pediatrics were excluded from further analysis.

\section{Travel time calculations}

Population centroids of each Census tract were mapped on a road network to the closest facility with at least one diplomate using the default settings of the Network Analyst tool in ArcMap (version 10.7.1) [18]. This was conducted annually between 2011 and 2019, inclusive. For each year, the median travel time for all Census tracts within a county was assigned as the county-level travel time.

\section{County obesity prevalence and covariate data}

Obesity prevalence was determined annually with the University of Wisconsin County Health Rankings and Roadmap dataset [19]. These values are derived from the Centers for Disease Control and Prevention Behavioral Risk Factor Surveillance System (CDC BRFSS) annual estimates based on self-reported height and weight data, which have been validated across other studies [20,21]. County-level sociodemographic data included the distribution of sex, race, ethnicity, education, and age as well as median household income and rurality. All covariates except for rurality were determined from the Census Bureau's American Community Survey; five-year estimates of annual data were chosen to precisely capture population estimates $[22,23]$. Age groups were combined to represent two distinct populations: adult (ages 18-64) and geriatric (ages 65 and above). Rurality was derived from the county-level Rural-Urban Continuum Codes classification out of the US Department of Agriculture and split into three categories (urban, suburban, and rural) based on National Cancer Institute (NCl) Surveillance, Epidemiology, and End Results Program guidelines $[24,25]$.

\section{Facility-level obesity diplomate availability}

To identify the distribution of diplomates per 100000 mapped patients with obesity, the following formula was developed:

$$
D P C_{h, y}=\frac{d_{h, y}}{\sum_{h, y, c=1, t=1}^{c=N_{c}, t=N_{t}} p_{t, h, y} O_{c, h, y}}
$$

where $N_{c}$ and $N_{t}$ denote the total numbers of counties and Census tracts present in any year in the data set. If in year $y$ Census tract $t$ is not mapped to facility $h$, then $p_{t, h, y}=0$. Overall, the denominator of the equation represents the total number of individuals with obesity who were mapped to facility $h$ in year $y$. This was calculated by multiplying the population $(p)$ of the mapped Census Tract $(t)$ for that year with the county (c)-level obesity prevalence (o) for the same year. This was then summed over all census tracks mapped to the facility $\left(\left(\sum_{h, y, c=1, t=1}^{c=N_{c}, t=N_{t}} p_{t, h, y} O_{c, h, y}\right)\right)$. The numerator of the equation represents the cumulative number of diplomates $(d)$ at facility $h$ between $2011(y=1)$ and the year of interest $(y=y)$. This includes diplomates certified in all prior years as well as newly certified diplomates. After division, the resulting value was multiplied by 100000 to get the number of diplomates per 100000 patients with obesity.

Three weight management patient-visit guidelines were selected to evaluate whether the supply of diplomates would be sufficient to treat the mapped patient populations. Those included the 2000 National Institutes of Health National Heart, Lung, and Blood Institute (NIH NHLBI) Obesity Education Initiative Guidelines (which suggests visits every four weeks for the first three months); the 2008 Centers for Medicare and Medicaid Services (CMS) Reimbursement Guidelines (which suggests weekly visits in the first month); and "usual care" from a 2011 randomized controlled trial by Wadden and colleagues ("Wadden et al.") on obesity treatment in primary care (which consisted of quarterly visits during the first 24 months of treatment) [26-28]. For these estimates, it was assumed that diplomates had $36 \mathrm{~h}$ a week for patient time based on a $40 \mathrm{~h}$ work week. This corresponded to $\sim 7.2$ hours of patient time per day or 36 to 72 unique patients per week assuming either all $1 \mathrm{~h}$ visits or all $30 \mathrm{~min}$ visits, respectively. It was also assumed that there were 48 work weeks in a year. The annual patient demand within each set of guidelines was calculated and compared to the available supply of diplomates to evaluate whether each facility met, exceeded, or fell below the recommended number of visits per patient (see eMethods 2 for a sample calculation).

\section{Statistical and sensitivity analysis}

County-level travel times and diplomates per capita were assessed with descriptive statistics (e.g., medians and interquartile ranges). The median travel time for different sociodemographic groups was calculated by weighting county travel times by the proportion of individuals within the group of interest that resided within that county. Intra- and inter-year variations in travel time between 2011 and 2019 were assessed with either population-weighted t-tests or population-weighted linear regression depending on whether the variable was dichotomous or categorical, respectively. For categorical tests, this resulted in a simultaneous test for variation across all levels of the predictor. A sensitivity analysis was conducted to evaluate how travel times for various sociodemographic groups varied when the underlying population was limited to just individuals with obesity (as opposed to all individuals); results for this analysis are presented in eTable 1.

Descriptive percentages were calculated to characterize the percent of facilities each year able to serve the estimated mapped population with obesity under the three clinical guidelines. A second sensitivity analysis reevaluated variations in facility capacity assuming only patients with severe obesity (i.e., BMI above $40 \mathrm{~kg} / \mathrm{m}^{2}$ ) regularly sought care from a diplomate. The percent of patients within a county with severe obesity was estimated from state-level data from the CDC BRFSS for all years except 2019, as data for New Jersey was unavailable [29]. Analyses were conducted with the statistical software R (version 3.6.3, Vienna, Austria) in the RStudio GUI (version 1.3.959, Boston, Massachusetts, USA).

\section{RESULTS}

There were 3036 diplomates included in the analysis (see eMethods 1 for the selection process and eFig. 1 for the distribution throughout the continental US). In 2011, the population-weighted median travel time to a diplomate was 28.5 minutes [IQR: 13.7, 68.1] (Fig. 1). An estimated $69.5 \%$ of Americans with obesity ( $n \sim 56.3$ million) lived within one hour of an obesity medicine diplomate, $26.9 \%$ ( $n \sim 21.7$ million) lived between 1 and $3 \mathrm{~h}$ from one, and 3.65\% ( $n \sim 2.96$ million) lived over three hours away from one. The median number of diplomates per 100000 mapped patients with obesity was estimated to be 1.4 [IQR: $0.39,1.59$ ]. In 2019, the populationweighted median travel time to a diplomate dropped to 9.95 minutes [IQR: 7.49, 18.1], which was significantly less than the 2011 value $(P<0.001$, Table 1). All sociodemographic groups (i.e., sex, age, race, ethnicity, education, median household income, rurality, and census region) had significantly shorter 


\section{Median County-Level Travel Time to Obesity Medicine Specialists}
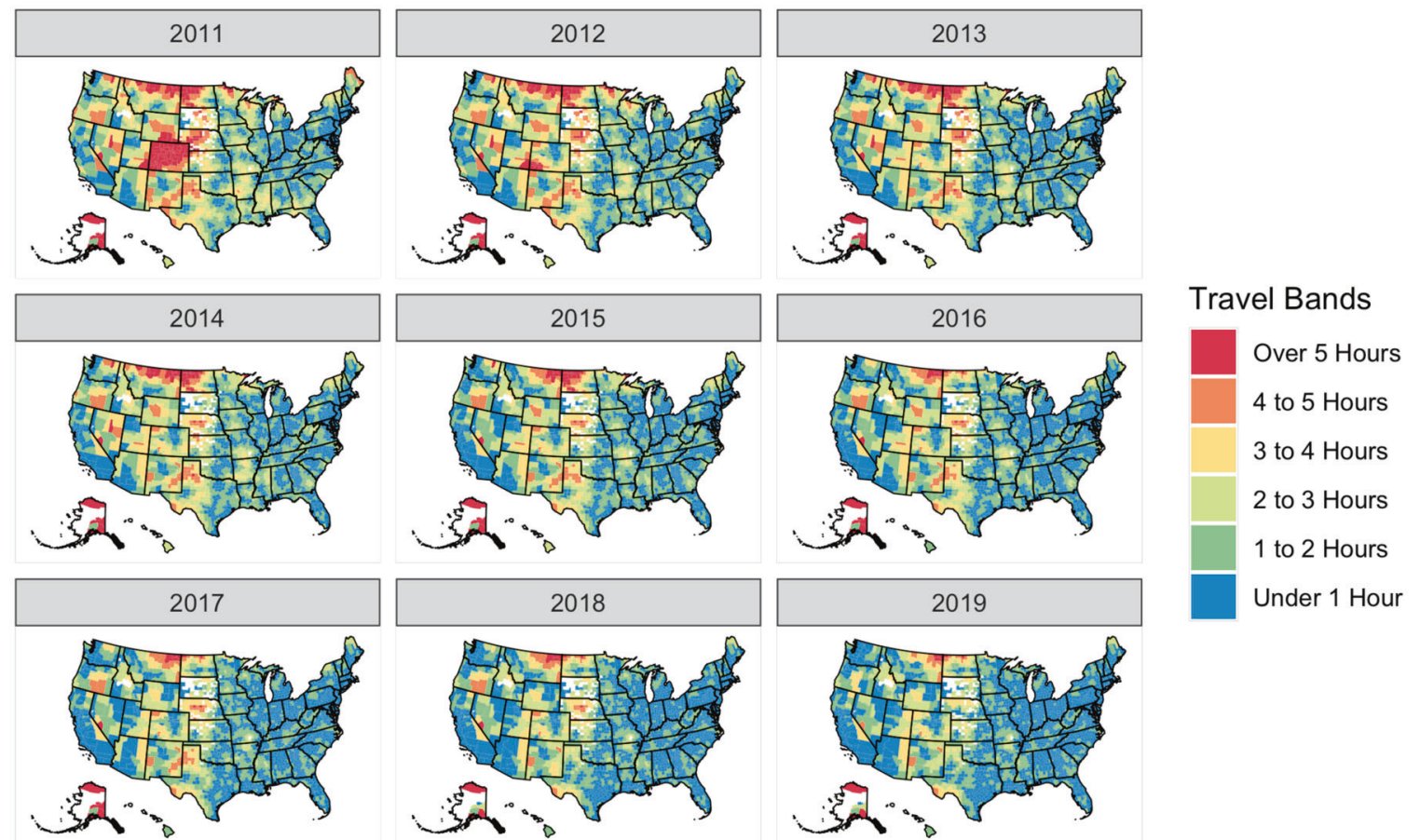

Fig. 1 Longitudinal changes in county-level travel time to obesity medicine diplomates. Travel time is defined as the median travel time of all population-weighted Census tracts within a county to the nearest facility on a road network with at least one obesity medicine diplomate.

travel times in 2019 compared to 2011 (all $P<0.001$ ). There were significant intra-year differences in both 2011 and 2019 by race, ethnicity, education, median household income, rurality, and Census region (all $P<0.001$ ). Adult and geriatric travel times were not significantly different in 2011, but they were in $2019\left(P_{2011}=\right.$ $0.14, P_{2019}=0.003$ ). Despite these improvements, the median number of diplomates per 100,000 mapped patients with obesity only rose to 5.06 diplomates [IQR: 2.74, 11.4] (eFig. 2).

Over time, the percent of facilities with an estimated capacity to serve the mapped patient population increased (Fig. 2). In 2019, under the oldest set of guidelines from the $\mathrm{NIH} \mathrm{NHLBI}$, approximately $0.52 \%$ of facilities could treat all mapped patients assuming 30 -minute-long visits, compared to $0.17 \%$ of facilities assuming one-hour-long visits. Using the CMS reimbursement guidelines from 2008, $0.12 \%$ of facilities in 2019 could treat all mapped patients assuming 30-minute-long visits, compared to $0.06 \%$ of facilities assuming one-hour-long visits. Applying the "usual care" guidelines set forth by the 2011 Wadden et al. study to $2019,1.73 \%$ of facilities could treat all patients assuming 30 min-long visits, compared to $0.87 \%$ of facilities assuming onehour-long visits. However, if only patients with severe obesity were assumed to need regular treatment from a diplomate, the estimated number of facilities that could accommodate mapped patients would rise to $0.69-1.63 \%$ of facilities based on NIH NHLBI guidelines; $0.09-0.19 \%$ of facilities based on CMS guidelines; and $3.43-8.68 \%$ of facilities based on the Wadden et al. study.

\section{DISCUSSION}

This study is the largest to date to comprehensively evaluate the longitudinal accessibility to obesity medicine diplomates in the US. The travel time to the nearest diplomate significantly decreased between 2011 and 2019, while the number of diplomates per 100000 mapped patients with obesity grew. However, only a small fraction of facilities was estimated to have adequate diplomates to accommodate all mapped patients by
2019 within the guidelines set forth by leading health experts. The results presented here demonstrate that improving physical access through decreased travel times is only part of the larger picture of health care accessibility, and considerations must also be made as to whether the number of providers can adequately serve the patient population in need.

While travel times have decreased significantly for all sociodemographic groups, disparities still exist. For example, almost every racial group evaluated in 2019 had travel times in the $75^{\text {th }}$ percentile that were not estimated to exceed $25 \mathrm{~min}$. The exception to this was American Indian and Alaska Native populations, whose interquartile range of travel time spanned from eight minutes to close to an hour. In addition, while the median travel time for counties in the highest quartile of income was $9 \mathrm{~min}$ in 2019, those residing in counties in the lowest quartile of income had a median travel time of $43 \mathrm{~min}$ to an obesity medicine diplomate. Stark differences also existed by rurality, with the median travel time for rural counties over $600 \%$ greater than that of urban counties. These disparities mirror findings from the field of oncology, which found disparities in access to NCl-Cancer Centers by race, rurality, income, and other sociodemographic characteristics [30]. One solution to rural access is telemedicine, which has seen an uptake in utilization throughout the COVID-19 pandemic due to temporary relaxations in interstate practicing law and improvements in reimbursement parity [31-33]. The benefits of telemedicine extend beyond rural communities, as non-Hispanic Black individuals with obesity may be more likely to utilize telemedicine than white individuals, although the same may not be true for other races and ethnicities [34]. Despite its promise, technological barriers to widespread telemedicine implementation (such as reduced broadband access and variations in technology literacy) remain, and thus geographic access remains of critical importance $[35,36]$. No matter the method to improve access, it is vital that these considerations include diplomates who practice in diverse communities, especially given the high prevalence of obesity (and severe obesity) within these groups [1, 37]. 
Table 1. Travel time variations by sociodemographic characteristics.

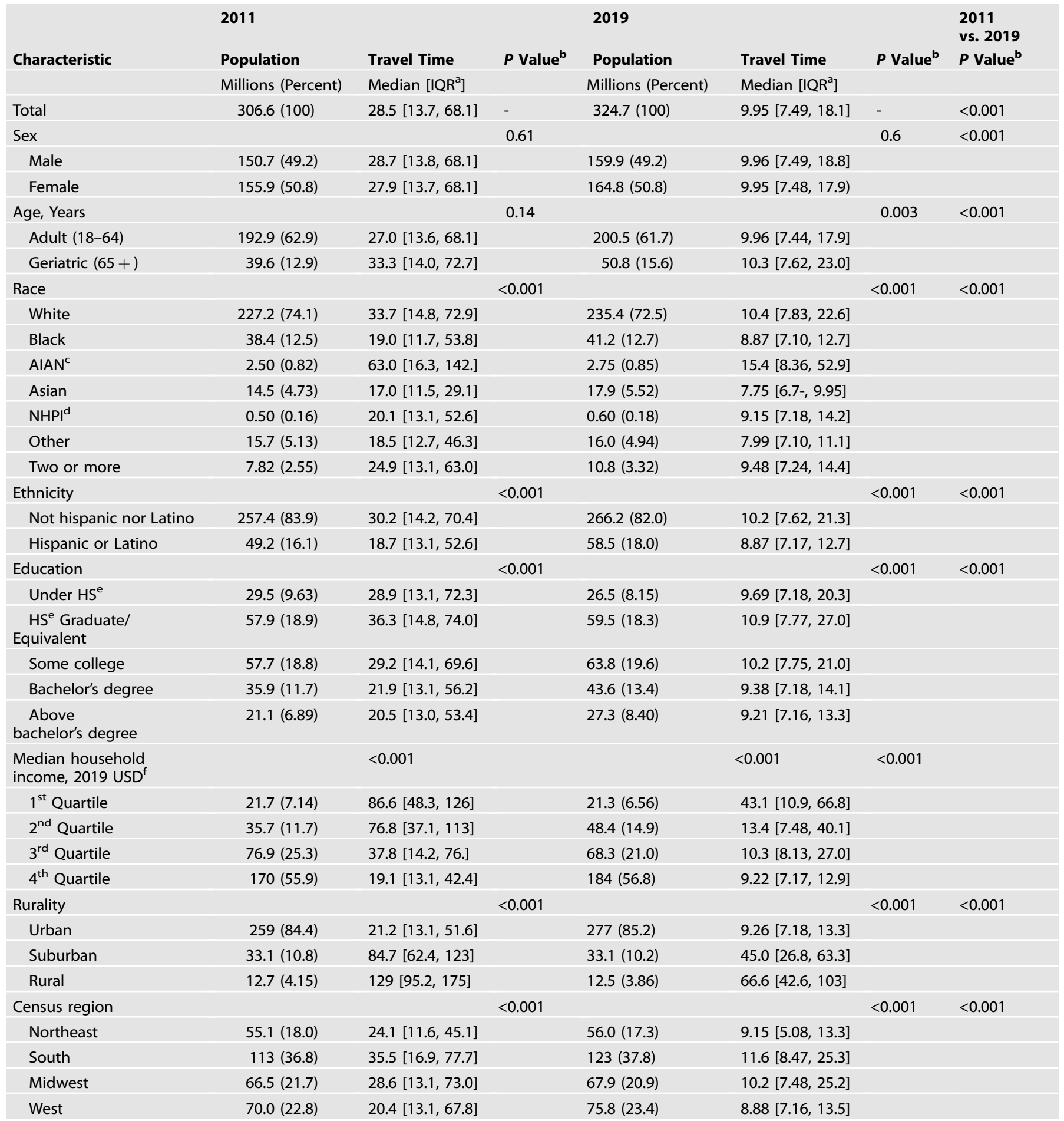

a $I Q R$ Interquartile Range.

${ }^{\mathrm{b} P}$-value evaluated from either weighted t-tests for dichotomous covariates or weighted linear regression for all others.

${ }^{\mathrm{C}}$ AIAN American Indian or Alaska Native.

${ }^{\mathrm{d} N H P I}$ Native Hawaiian or Pacific Islander.

eHS High School.

fUSD US Dollars. Quartiles were defined as follows: 2011 Q1: $\$ 22,306-\$ 43,018 ; 2011$ Q2: \$43,018-\$49,418; 2011 Q3: \$49,418-\$57,160; 2011 Q4: \$57,160\$137,080; 2019 Q1: $\$ 21,504-\$ 44,114 ; 2019$ Q2: \$44,114-\$51,659; 2019 Q3: \$51,659-\$59,734; 2019 Q4: \$59,734-\$142,299. 
Percent of Facilities that have Capacity to Serve the Mapped Patient Population

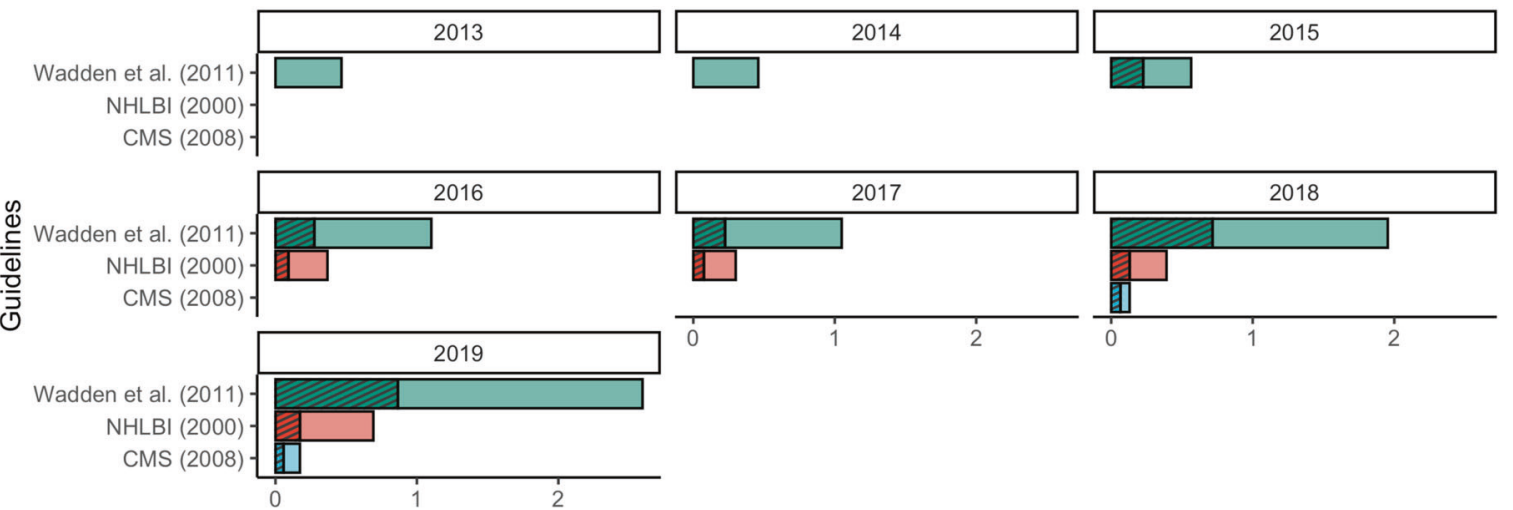

Percent (\%)

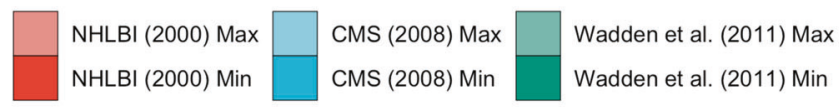

$\operatorname{Max}$ or Min $\square \operatorname{Max} / / \operatorname{Min}$

Fig. 2 Percent of facilities with capacity to serve the mapped patient population. Darker shades and hatches for the National Heart, Lung and Blood Institute (NHLBI), Centers for Medicare and Medicaid Services (CMS), and Wadden et al. studies denote the minimum number of facilities assuming all patients received 1-hour visits, while lighter shades for those recommendations denote the maximum number of facilities assuming all patients received 30-minute visits.

Further, the median number of diplomates per 100000 mapped patients with obesity remains strikingly low, despite the fact that the number of facilities that are estimated to be able to serve their patient population within a set of recommendations rose from $0 \%$ in 2011 to a maximum of $1.73 \%$ of facilities in 2019 under "usual care" guidelines established by Wadden et al. [28]. This is still likely an overestimation, as the majority of diplomates do not spend their entire workday practicing obesity medicine -- a study in 2016 found that, while over 2000 clinicians were certified, only $30.8 \%$ spent at least half of their practice time on obesity medicine [38]. This implies that patients with obesity who want to receive care may be limited in their ability to actually receive the care, even if they are located proximately to a diplomate or able to utilize mobile health technologies (such as telemedicine) for a consultation. Even if it is assumed that only patients with severe obesity require specialty care, only a maximum of $8.68 \%$ of facilities have the capacity to serve the nearest population in need. This suggests that patients may encounter exceedingly long wait times when trying to receive care that may deter their desire or inhibit their ability to receive care altogether.

It is important to consider whether every patient with obesity necessarily needs to be treated by an obesity medicine diplomate. Given the low number of diplomates per 100000 mapped patients with obesity, it may be more feasible if only patients with severe obesity regularly consulted with diplomates. Yet, given the high prevalence of obesity in the US, increasing the amount of training that all providers receive in obesity medicine could help to ensure that patients with obesity can be informed of the potential treatment options available to them. It is possible that obesity medicine could benefit from a model like Project Extension for Community Healthcare Outcomes (ECHO) whereby certified diplomates could help train other physicians within their practice in order to create a network of expertise [39]. Future work is needed to evaluate whether this type of provider network model could be effectively applied to facilitate obesity medicine education within and across diverse facilities. Along the same lines, it is vital to explore how variations in obesity medicine education among diplomates may impact practice patterns. The ABOM has two primary pathways for certification: one that requires a fellowship, and one that relies on CME credit. As a result, it may be valuable to explore the geographic distribution and outcomes of diplomates by pathway type. However, such an analysis may be challenging as fewer than $5 \%$ of diplomates with available information have applied through the fellowship pathway (which includes fellowships in either obesity medicine or another specialty with a substantial portion on obesity medicine, such as endocrinology or bariatric surgery) [40].

While this study is specific to the US, understanding access to physicians with specialized training in obesity medicine is of critical international importance. A recent systematic review that included studies from the US, United Kingdom, and Canada found a dearth of training programs in obesity medicine [7]. Given the global rise in obesity prevalence, other countries would also likely benefit from increased access to providers with obesity medicine training [41]. The benefits of such educational opportunities are vast given their coverage of key topics such as the mitigation of weight bias. This may be especially important as a study in the US found that perceived judgement from a healthcare provider was inversely associated with both attempted and successful weight loss [42]. Thus, additional educational opportunities that span a variety of cultures could be a valuable tool to reduce the stigmatization of patients with obesity and improve health outcomes.

This study is not without its limitations. The results presented here only relate to adult obesity, and future analyses could repeat this process with a pediatric population. Patient locations were estimated using population-weighted Census tracts, which do not directly correlate to patient addresses. While differences may be minimal in urban communities, using an estimated location may be more problematic in rural communities with a sparse population. Thus, future work could clarify the extent of variation in travel time estimates when precise patient addresses are used in place of population-weighted geographic approximations. Further, facility mapping assumed that all patients would visit their nearest facility, which may not necessarily be the case. However, in one study on access to mammography facilities, the majority of patients chose to use either their closest facility or one within five minutes of the closest facility, providing a logical basis for mapping patients to their 
nearest facility [43]. Future studies could provide more constraints on the mapping, such as the number of patients mapped to each facility. Public transportation methods and traffic patterns may also be considered in future work. Additionally, due to the ecological nature of the study, no conclusions can directly be drawn to any individual patient. The study does not capture how many persons with obesity sought care from a diplomate, nor does it evaluate the impact of a diplomate deficit on the desire to seek care. Similarly, the estimated amount of time that diplomates spent on patients per week (i.e., $36 \mathrm{~h}$ ) is likely an overestimation of availability, especially given highly variable inter-practice patterns. Thus, the estimates presented here are likely an underestimation of clinics' capacities to serve a mapped theoretical patient population. Therefore, future work should directly measure the number of time diplomates spend on patients per week and whether access to a diplomate is associated with the receipt of care and health outcomes. Also, this study assumed that a diplomate's practicing location was static and accurately listed online. Follow-up studies are needed to assess whether this assumption holds. Also, the guidelines from the $\mathrm{NIH}$ NHLBI may be outdated, and future work could apply this analysis to alternative, more recent guidelines. Finally, given the increasingly widespread implementation of telemedicine (particularly for rural clinics), future estimates of diplomate care burden should incorporate this mechanism of care delivery.

In summary, access to obesity medicine diplomates has greatly improved over time as measured through both geographic access and provider availability. While the number of diplomates per 100000 mapped patients with obesity and the number of facilities able to successfully treat all mapped patients within specified guidelines has increased, there still exists a limited number of facilities that can help patients achieve sustainable weight loss and improvements in corresponding clinical outcomes. This is particularly salient as facilities increase their telemedicine capabilities and enhance accessibility to these services - whether access to obesity medicine diplomates is virtual or physical, there are not enough to adequately serve all patients. Health policymakers should develop strategies that focus on how to provide additional training for physicians in obesity medicine, be it through encouraging providers to pursue ABOM certification, enhancing the medical school curriculum to include more obesity content, or leveraging an ECHO-like model to generate an interconnected network of interdisciplinary providers.

\section{CODE AVAILABILITY}

The final, curated data sets are available upon request. Code can be accessed at https://github.com/cpollack736/ObesityCertification_ABOM.

\section{REFERENCES}

1. Hales CM, Carroll MD, Fryar CD, Ogden CL Prevalence of Obesity and Severe Obesity Among Adults: United States, 2017-2018. National Center for Health Statistics: Hyattsville, MD, 2020 https://www.cdc.gov/nchs/products/databriefs/ db360.htm (accessed 5 Nov 2020).

2. Diabetes Prevention Program Research Group. Reduction in the incidence of type 2 diabetes with lifestyle intervention or metformin. England J Med. 2009;346:393-403.

3. Wing RR, Lang W, Wadden TA, Safford M, Knowler WC, Bertoni AG, et al. Benefits of modest weight loss in improving cardiovascular risk factors in overweight and obese individuals with type 2 diabetes. Diabetes Care. 2011;34:1481-6.

4. Pischon T, Nöthlings $U$, Boeing $H$. Obesity and cancer. Proc Nutr Soc. 2008;67:128-45.

5. Richardson S, Hirsch JS, Narasimhan M, Crawford JM, McGinn T, Davidson KW, et al. Presenting characteristics, comorbidities, and outcomes among 5700 patients hospitalized with COVID-19 in the New York City area. JAMA. 2020;323:2052.

6. Kushner RF, Butsch WS, Kahan S, Machineni S, Cook S, Aronne LJ. Obesity coverage on medical licensing examinations in the United States. What is being tested? Teach Learn Med. 2017;29:123-8.

7. Mastrocola MR, Roque SS, Benning LV, Stanford FC. Obesity education in medical schools, residencies, and fellowships throughout the world: A systematic review. Int J Obes. 2020;44:269-79.
8. Butsch WS, Kushner RF, Alford S, Smolarz BG. Low priority of obesity education leads to lack of medical students' preparedness to effectively treat patients with obesity: Results from the U.S. medical school obesity education curriculum benchmark study. BMC Med Educ. 2020;20:23.

9. Yarlagadda S, Townsend MJ, Palad CJ, Stanford FC. Coverage of obesity and obesity disparities on American Board of Medical Specialties (ABMS) examinations. J Natl Med Assoc. 2021;S0027-9684(21)00051-1.

10. CME Certification Pathway Eligibility and Requirements. American Board Obes Med. https://www.abom.org/cme-certification-pathway-eligibility-and-requirements/ (accessed 6 Apr 2021).

11. Eligibility: Fellowship Pathway. American Board Obes Med. https://www.abom. org/eligibility-fellowship-pathway/ (accessed 6 Apr 2021).

12. Exam Resources 2. American Board Obes Med. https://www.abom.org/examresources-2/ (accessed 6 Apr 2021).

13. Alexander L. The benefits of obesity medicine certification. Am J Lifestyle Med. 2018;13:161-4.

14. Tsai AG, Histon T, Kyle TK, Rubenstein N, Donahoo WT. Evidence of a gap in understanding obesity among physicians. Obes Sci Pract. 2018;4:46-51.

15. Gudzune KA, Wickham EP, Schmidt SL, Stanford FC. Physicians certified by the American board of obesity medicine provide evidence-based care. Clin Obes. 2021;11:e12407.

16. Gudzune KA, Johnson VR, Bramante CT, Stanford FC. Geographic availability of physicians certified by the american board of obesity medicine relative to obesity prevalence. Obesity. 2019;27:1958-66.

17. [LB: ABOM] Diplomate Search and Certification Verification. https://abom. learningbuilder.com/public/membersearch (accessed 24 Sep 2020).

18. Geography Division, US Census Bureau. Centers of Population Computation for the United States: 1950-2010. US Department of Commerce: Washington, DC, 2011.

19. How Healthy is your County? | County Health Rankings. County Health Rankings \& Roadmaps. https://www.countyhealthrankings.org/county-health-rankingsroadmaps (accessed 24 Sep 2020).

20. Ewing R, Schmid T, Killingsworth R, Zlot A, Raudenbush S. Relationship between Urban sprawl and physical activity, obesity, and morbidity. Am J Health Promot. 2003;18:47-57.

21. Sturm R, Hattori A. Morbid obesity rates continue to rise rapidly in the United States. Int J Obes. 2013;37:889-91.

22. Bureau UC. About the Survey. The United States Census Bureau. https://www. census.gov/programs-surveys/acs/about.html (accessed 24 Sep 2020).

23. Bureau UC. When to Use 1-year, 3-year, or 5-year Estimates. The United States Census Bureau. https://www.census.gov/programs-surveys/acs/guidance/ estimates.html (accessed 6 Apr 2021).

24. USDA ERS - Rural-Urban Continuum Codes. https://www.ers.usda.gov/dataproducts/rural-urban-continuum-codes.aspx (accessed 24 Sep 2020).

25. Rural-Urban Continuum Code - SEER Datasets. SEER. https://seer.cancer.gov/ seerstat/variables/countyattribs/ruralurban.html (accessed 24 Sep 2020).

26. The Practica Guide for Identification, Evaluation, and Treatment of Overweight and Obesity in Adults. National Heart, Lung, and Blood Institute: Bethesda, MD, 2000.

27. Fitzpatrick SL, Wischenka D, Appelhans BM, Pbert L, Wang M, Wilson DK, et al. An evidence-based guide for obesity treatment in primary care. Am J Med. 2016;129:115.e1-115.e7.

28. Wadden TA, Volger S, Sarwer DB, Vetter ML, Tsai AG, Berkowitz RI, et al. A twoyear randomized trial of obesity treatment in primary care practice. New England J Med. 2011;365:1969-79.

29. CDC - 2019 BRFSS Survey Data and Documentation. 2020. https://www.cdc.gov/ brfss/annual_data/annual_2019.html (accessed 6 Apr 2021).

30. Onega T, Duell EJ, Shi X, Wang D, Demidenko E, Goodman D. Geographic access to cancer care in the U.S. Cancer. 2008:112:909-18.

31. Hollander JE, Carr BG Virtually Perfect? Telemedicine for Covid-19. New England J Med 2020; 382: 1679-81.

32. Trump Administration Drives Telehealth Services in Medicaid and Medicare. Centers for Medicare \& Medicaid Services. 2020. https://www.cms.gov/ newsroom/press-releases/trump-administration-drives-telehealth-servicesmedicaid-and-medicare (accessed 18 Dec 2020).

33. Patel SY, Mehrotra A, Huskamp HA, Uscher-Pines L, Ganguli I, Barnett ML. Trends in outpatient care delivery and telemedicine during the COVID-19 pandemic in the US. JAMA Intern Med. 2021;181:388.

34. Almandoz JP, Xie L, Schellinger JN, Mathew MS, Edwards K, Ofori A et al. Telehealth utilization among multi-ethnic patients with obesity during the COVID-19 pandemic. J Telemed Telecare. 2021; 1357633X21998211.

35. Koonin LM. Trends in the Use of Telehealth During the Emergence of the COVID19 Pandemic - United States, January-March 2020. MMWR Morb Mortal Wkly Rep. 2020;69. https://doi.org/10.15585/mmwr.mm6943a3.

36. Chen J, Amaize A, Barath D. Evaluating Telehealth Adoption and Related Barriers Among Hospitals Located in Rural and Urban Areas. J Rural Health; n/a. https:// doi.org/10.1111/jrh.12534. 
37. Centers for Disease Control and Prevention. CDC and Indian country: Working together. centers for disease control and prevention, US Dept. of Health and Human Services: Atlanta, Georgia, 2017.

38. Kushner RF, Brittan D, Cleek J, Hes D, English W, Kahan S, et al. The American board of obesity medicine: Five-year report. Obesity. 2017;25:982-4.

39. Arora S, Kalishman S, Dion D, Som D, Thornton K, Bankhurst A, et al. Partnering urban academic medical centers and rural primary care clinicians to provide complex chronic disease care. Health Aff (Millwood). 2011;30:1176-84.

40. Burke J. (American Board of Obesity Medicine, Denver, CO). Email to: Catherine Pollack (Geisel School of Medicine at Dartmouth College, Lebanon, NH). 2021

41. Malik VS, Willet WC, Hu FB. Nearly a decade on - trends, risk factors and policy implications in global obesity. Nat Rev Endocrinol. 2020;16:615-6.

42. Gudzune KA, Bennett WL, Cooper LA, Bleich SN. Perceived judgment about weight can negatively influence weight loss: A cross-sectional study of overweight and obese patients. Prev Med. 2014;62:103-7.

43. Alford-Teaster J, Lange JM, Hubbard RA, Lee Cl, Haas JS, Shi X, et al. Is the closest facility the one actually used? An assessment of travel time estimation based on mammography facilities. Int J Health Geogr. 2016;15:8.

\section{ACKNOWLEDGEMENTS}

The authors would like to acknowledge Jennifer Alford-Teaster and Stephen Gaughan for their technical support and suggestions regarding the geographic analysis in ArcMap.

\section{AUTHOR CONTRIBUTIONS}

CCP, DGD, and TO formulated the research question; all authors designed the analysis; CCP, DGD, and TO analyzed the data; CCP, DGD, and TO drafted the initial paper; all authors revised and approved the final manuscript.

\section{COMPETING INTERESTS}

This publication was supported in part by an NLM-funded predoctoral fellowship to CCP (5T32LM012204-05). Its contents are solely the responsibility of the authors and do not represent the official views of the NLM or NIH.

\section{ADDITIONAL INFORMATION}

Supplementary information The online version contains supplementary material available at https://doi.org/10.1038/s41366-021-01024-9.

Correspondence and requests for materials should be addressed to Catherine $C$. Pollack.

Reprints and permission information is available at http://www.nature.com/ reprints

Publisher's note Springer Nature remains neutral with regard to jurisdictional claims in published maps and institutional affiliations. 